\title{
MULTIVALUED $H$-ESSENTIAL MAPS OF ACYCLIC TYPE ON HAUSDORFF TOPOLOGICAL SPACES
}

\author{
DONAL O'REGAN
}

Received 7 March 2005; Revised 25 April 2005; Accepted 28 April 2005

This paper discusses acyclic maps between topological spaces. We show that if $F$ is $H$ essential and $F \cong G$, then $G$ has a fixed point.

Copyright (C) 2006 Donal O’Regan. This is an open access article distributed under the Creative Commons Attribution License, which permits unrestricted use, distribution, and reproduction in any medium, provided the original work is properly cited.

\section{Introduction}

The notion of an essential map in a Banach (or Fréchet) space setting introduced by Granas in [2] is more general than the notion of degree. In [2] he showed that if $F$ is essential and $F \cong G$, then $G$ is essential. However to be essential is quite general and as a result Granas was only able to show this homotopy property for particular classes of maps (the most general being compact (or condensing) Kakutani maps). However from an application viewpoint, one does not need such a strong homotopy property. In fact one usually only needs that if $F$ is essential and $F \cong G$, then $G$ has a fixed point. In this paper we establish this result for a large class of maps (namely, the acyclic maps). An added bonus is that the result holds for maps between Hausdorff topological spaces (i.e., the spaces need not be vector spaces).

Let $X$ and $Z$ be subsets of Hausdorff topological spaces. We will consider maps $F: X \rightarrow$ $K(Z)$; here $K(Z)$ denotes the family of nonempty compact subsets of $Z$. A nonempty topological space is said to be acyclic if all its reduced Cech homology groups over the rationals are trivial. Now $F: X \rightarrow K(Z)$ is acyclic if $F$ is upper semicontinuous with acyclic values.

\section{Continuation theory}

Throughout this section $Y$ will be a completely regular topological space and $U$ will be an open subset of $Y$.

Definition 2.1. It is said that $F \in A C(\bar{U}, Y)$ if $F: \bar{U} \rightarrow K(Y)$ is an acyclic compact map; here $\bar{U}$ denotes the closure of $U$ in $Y$. 
Definition 2.2. It is said that $F \in A C_{\partial U}(\bar{U}, Y)$ if $F \in A C(\bar{U}, Y)$ with $x \notin F(x)$ for $x \in \partial U$; here $\partial U$ denotes the boundary of $U$ in $Y$.

Definition 2.3. Let $F, G \in A C_{\partial U}(\bar{U}, Y)$. It is said that $F \cong G$ in $A C_{\partial U}(\bar{U}, Y)$ if there exists a upper semicontinuous compact map $\Psi: \bar{U} \times[0,1] \rightarrow K(Y)$ with $\Psi_{t} \in A C_{\partial U}(\bar{U}, Y)$ for each $t \in[0,1], \Psi_{1}=F$ and $\Psi_{0}=G\left(\right.$ here $\left.\Psi_{t}(x)=\Psi(t, x)\right)$.

Definition 2.4. A map $F \in A C_{\partial U}(\bar{U}, Y)$ is said to be $H$-essential in $A C_{\partial U}(\bar{U}, Y)$ if every map $G \in A C_{\partial U}(\bar{U}, Y)$ with $\left.G\right|_{\partial U}=\left.F\right|_{\partial U}$ and $G \cong F$ in $A C_{\partial U}(\bar{U}, Y)$ has a fixed point in $U$.

We immediately present our continuation theorem.

THeORem 2.5. Let $Y$ be a completely regular topological space, let $U$ be an open subset of $Y$, and let $F \in A C_{\partial U}(\bar{U}, Y)$ be $H$-essential in $A C_{\partial U}(\bar{U}, Y)$. Suppose there exists an upper semicontinuous compact map $H: \bar{U} \times[0,1] \rightarrow K(Y)$ with $H_{t} \in A C(\bar{U}, Y)$ for each $t \in[0,1]$, $H_{0}=F$ and $x \notin H_{t}(x)$ for $x \in \partial U$ and $t \in(0,1]$. Then $H_{1}$ has a fixed point in $U$.

Proof. Let

$$
B=\left\{x \in \bar{U}: x \in H_{t}(x) \text { for some } t \in[0,1]\right\} .
$$

Notice that $B \neq \varnothing$ since $F$ is $H$-essential in $A C_{\partial U}(\bar{U}, Y)$ (note in particular that $F \cong F$ in $A C_{\partial U}(\bar{U}, Y)$ and so $F$ has a fixed point in $U$ ). Also $B$ is closed (note that $H$ is upper semicontinuous) and in fact compact (note that $H$ is compact). Also note that $x \notin H_{t}(x)$ for $x \in \partial U$ and $t \in[0,1]$ (note that $H_{0}=F \in A C_{\partial U}(\bar{U}, Y)$ ) so $B \cap \partial U=\varnothing$. Now, since $Y$ is completely regular, there exists a continuous map $\mu: \bar{U} \rightarrow[0,1]$ with $\mu(B)=1$ and $\mu(\partial U)=0$. Define a map $R: \bar{U} \rightarrow K(Y)$ by

$$
R(x)=H(x, \mu(x))
$$

Clearly $R \in A C_{\partial U}(\bar{U}, Y)$ since $\left.R\right|_{\partial U}=\left.H_{0}\right|_{\partial U}=\left.F\right|_{\partial U}$. We claim that

$$
R \cong F \quad \text { in } A C_{\partial U}(\bar{U}, Y)
$$

If (2.3) is true, then, since $F$ is $H$-essential in $A C_{\partial U}(\bar{U}, Y)$, there exists $x \in U$ with $x \in$ $R(x)=H(x, \mu(x))$. Thus $x \in B$ so $\mu(x)=1$ and consequently $x \in H(x, 1)=H_{1}(x)$, and we are finished.

It remains to check (2.3). Let $Q: \bar{U} \times[0,1] \rightarrow K(Y)$ be given by

$$
Q(x, t)=H(x, t \mu(x))
$$

Now $Q_{0}=H_{0}=F$ and $Q_{1}(x)=H(x, \mu(x))=R(x)$, and clearly $Q: \bar{U} \times[0,1] \rightarrow K(Y)$ is an upper semicontinuous compact map with $Q_{t} \in A C(\bar{U}, Y)$ for each $t \in[0,1]$. Also $Q_{t}$ is fixed point free on $\partial U$ for each $t \in[0,1]$ since if there exists $t \in[0,1]$ and $x \in \partial U$ with $x \in Q_{t}(x)$, then $x \in H(x, t \mu(x))$, so $x \in B$, and as a result $\mu(x)=1$, that is, $x \in H(x, t)$, a contradiction. Thus (2.3) holds. 
We may now apply Theorem 2.5 to establish the main result in [1].

Definition 2.6. It is said that $F \in A C(Y, Y)$ if $F: Y \rightarrow K(Y)$ is an acyclic compact map.

Definition 2.7. If $F \in A C(Y, Y)$ and $p \in Y$ then it is said that $F \cong\{p\}$ in $A C(Y, Y)$ if there exists an upper semicontinuous compact map $R: Y \times[0,1] \rightarrow K(Y)$ with $R_{t} \in A C(Y, Y)$ for each $t \in[0,1], R_{1}=F$, and $R_{0}=\{p\}$ (here $R_{t}(x)=R(x, t)$ ).

THEOREM 2.8. Let $Y$ be a completely regular topological space, $U$ an open subset of $Y$, and $u_{0} \in U$. Suppose there exists an upper semicontinuous compact map $H: \bar{U} \times[0,1] \rightarrow K(Y)$ with $H_{t} \in A C(\bar{U}, Y)$ for each $t \in[0,1], H_{0}=\left\{u_{0}\right\}$ and with $x \notin H_{t}(x)$ for $x \in \partial U$ and $t \in(0,1]$. In addition, assume the following property holds:

$$
\begin{array}{r}
\text { for any } \Phi \in A C(Y, Y) \text { and any } p \in Y \text { with } \Phi \cong\{p\} \\
\text { in } A C(Y, Y), \Phi \text { has a fixed point in } Y .
\end{array}
$$

Then $H_{1}$ has a fixed point in $U$.

Proof. Let $F(x)=\left\{u_{0}\right\}$ for each $x \in \bar{U}$. The result follows from Theorem 2.5 if we show that $F \in A C_{\partial U}(\bar{U}, Y)$ is $H$-essential in $A C_{\partial U}(\bar{U}, Y)$. Certainly $F \in A C_{\partial U}(\bar{U}, Y)$ since $u_{0} \in$ $U$. Now let $G \in A C_{\partial U}(\bar{U}, Y)$ be any map with $\left.G\right|_{\partial U}=\left.F\right|_{\partial U}$ and $G \cong\left\{u_{0}\right\}$ in $A C_{\partial U}(\bar{U}, Y)$. To show that $F$ is $H$-essential in $A C_{\partial U}(\bar{U}, Y)$, we must show that $G$ has a fixed point in $U$.

We know there exists an upper semicontinuous compact map $\Lambda: \bar{U} \times[0,1] \rightarrow K(Y)$ with $\Lambda_{t} \in A C_{\partial U}(\bar{U}, Y)$ for each $t \in[0,1], \Lambda_{0}=\left\{u_{0}\right\}$ and $\Lambda_{1}=G$. Now let

$$
D=\left\{x \in \bar{U}: x \in \Lambda_{t}(x) \text { for some } t \in[0,1]\right\} .
$$

Notice that $D \neq \varnothing\left(\right.$ since $\left.u_{0} \in U\right)$ is closed and compact and $D \cap(Y \backslash U)=\varnothing$. Thus there exists a continuous map $\sigma: Y \rightarrow[0,1]$ with $\sigma(D)=1$ and $\sigma(Y \backslash U)=0$. Define $\Psi: Y \times$ $[0,1] \rightarrow K(Y)$ by

$$
\Psi(x, t)= \begin{cases}\Lambda(x, t \sigma(x)), & x \in \bar{U} \\ \left\{u_{0}\right\}, & x \in Y \backslash U .\end{cases}
$$

Clearly $\Psi: Y \times[0,1] \rightarrow K(Y)$ is an upper semicontinuous compact map with $\Psi_{t} \in A C(Y$, $Y)$ for each $t \in[0,1]$ and as a result $\Psi_{1} \cong\left\{u_{0}\right\}$ in $A C(Y, Y)$. Now (2.5) guarantees that there exists $x \in Y$ with $x \in \Psi_{1}(x)$. If $x \in Y \backslash \bar{U}$ then $x \in\left\{u_{0}\right\}$, which is a contradiction since $u_{0} \in U$. Thus $x \in U$ so $x \in \Lambda(x, \sigma(x))$, and as a result $x \in D$, which implies $\sigma(x)=1$ and so $x \in \Lambda(x, 1)=G(x)$.

Remark 2.9. Condition (2.5) was discussed in [1] and we refer the reader to that paper. For the convenience of the reader we present some examples here. Suppose $X$ and $Z$ are topological spaces. Given a class $\mathscr{X}$ of maps, $\mathscr{X}(X, Z)$ denotes the set of maps $F: X \rightarrow 2^{Z}$ (nonempty subsets of $Z$ ) belonging to $\mathscr{X}$, and $\mathscr{X}_{c}$ the set of finite compositions of maps in $\mathscr{X}$. We let

$$
\mathscr{F}(\mathscr{X})=\{W: \operatorname{Fix} F \neq \varnothing \forall F \in \mathscr{L}(W, W)\},
$$

where Fix $F$ denotes the set of fixed points of $F$. 
The class $\mathcal{U}$ of maps is defined by the following properties:

(i) $\mathcal{U}$ contains the class $\mathscr{b}$ of single-valued continuous functions;

(ii) each $F \in U_{c}$ is upper semicontinuous and compact valued;

(iii) $B^{n} \in \mathscr{F}\left(U_{c}\right)$ for all $n \in\{1,2, \ldots\}$; here $B^{n}=\left\{x \in \mathbb{R}^{n}:|x| \mid \leq 1\right\}$.

Next we consider the class $u_{c}^{\kappa}(X, Z)$ of maps $F: X \rightarrow 2^{Z}$ such that for each $F$ and each nonempty compact subset $K$ of $X$ there exists a map $G \in U_{c}(K, Z)$ such that $G(x) \subseteq F(x)$ for all $x \in K$. By a space we mean a Hausdorff topological space. Let $Q$ be a class of topological spaces. A space $Y$ is an extension space for $Q$ (written $Y \in \operatorname{ES}(Q)$ ) if for all $X \in$ $Q$, for all $K \subseteq X$ closed in $X$, any continuous function $f_{0}: K \rightarrow Y$ extends to a continuous function $f: X \rightarrow Y$. Recall that if $X \in \mathrm{ES}$ (compact) and $F \in \mathcal{U}_{c}^{\kappa}(X, X)$, a compact map, then $F$ has a fixed point. Consequently we have the following result: if $Y \in \mathrm{ES}$ (compact) then any map $G \in A C(Y, Y)$ has a fixed point, so trivially (2.5) holds (notice that the condition that $G \cong\{p\}$ in $A C(Y, Y)$ in (2.5) does not play any role in this example).

For our next example we suppose $Y$ is a metrizable ANR. We claim that (2.5) holds. To see this let $G \in A C(Y, Y)$, let $p \in Y$, and let $G \cong\{p\}$ in $A C(Y, Y)$ (i.e., there exists an upper semicontinuous compact map $R: Y \times[0,1] \rightarrow K(Y)$ with $R_{t} \in A C(Y, Y)$ for each $t \in[0,1], R_{1}=G$, and $\left.R_{0}=\{p\}\right)$. By the Arens-Eells theorem we know that $Y$ can be regarded as a closed subset of a normed space $E$ (recall that the Arens-Eells theorem states that any metric space can be isometrically embedded as a closed subset in a normed linear space). Since $Y \in$ ANR, there is an open neighborhood $V$ of $Y$ in $E$ and a retraction $r: \bar{V} \rightarrow Y$. Let $\lambda: E \rightarrow[0,1]$ be a continuous function with $\lambda(E \backslash V)=0$ and $\lambda(Y)=1$. Also let

$$
Q(x)= \begin{cases}R(r(x), \lambda(x)), & x \in \bar{V}, \\ \{p\}, & x \in E \backslash V\end{cases}
$$

(note that if $x \in \partial V$ then $Q(x)=R(x, \lambda(x))=R(x, 0)=\{p\})$. Clearly for each $x \in E$ we have that $Q(x)$ is acyclic valued and it is easy to see that $Q: E \rightarrow K(E)$ is an upper semicontinuous compact map. Thus $Q \in A C(E, E)$. A well-known result guarantees that there exists $x_{0} \in E$ with $x_{0} \in Q\left(x_{0}\right)$. Notice that if $x_{0} \in E \backslash V$ then $x_{0} \in\{p\}$, which is a contradiction since $p \in Y$ (note $Y \subset V$ ). If $x_{0} \in \bar{V} \backslash Y$ then since $Q: E \rightarrow K(Y)$ (note that $R: Y \times[0,1] \rightarrow K(Y))$ and $x_{0} \in Q\left(x_{0}\right)$, we have that $x_{0} \in Y$, a contradiction. As a result $x_{0} \in Y$ so $r\left(x_{0}\right)=x_{0}, \lambda\left(x_{0}\right)=1$, and so $x_{0} \in R\left(x_{0}, 1\right)=G\left(x_{0}\right)$, that is, (2.5) holds.

\section{References}

[1] R. P. Agarwal, J. H. Dshalalow, and D. O'Regan, Generalized Leray-Schauder principles in Hausdorff topological spaces for acyclic maps, Mathematical Sciences Research Journal. An International Journal of Rapid Publication 8 (2004), no. 4, 114-117.

[2] A. Granas, Sur la méthode de continuité de Poincaré, Comptes Rendus de l'Académie des Sciences. Paris Séries A-B 282 (1976), no. 17, 983-985 (French).

Donal O'Regan: Department of Mathematics, National University of Ireland, Galway, Ireland E-mail address: donal.oregan@nuigalway.ie 


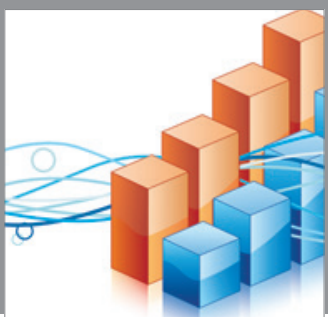

Advances in

Operations Research

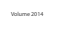

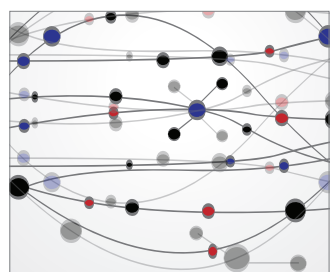

\section{The Scientific} World Journal
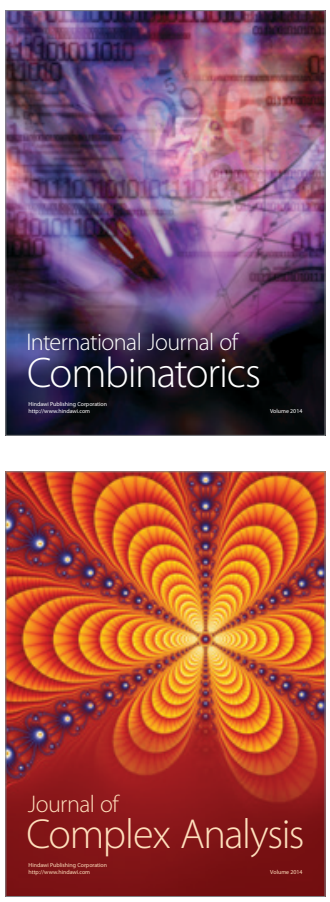

International Journal of

Mathematics and

Mathematical

Sciences
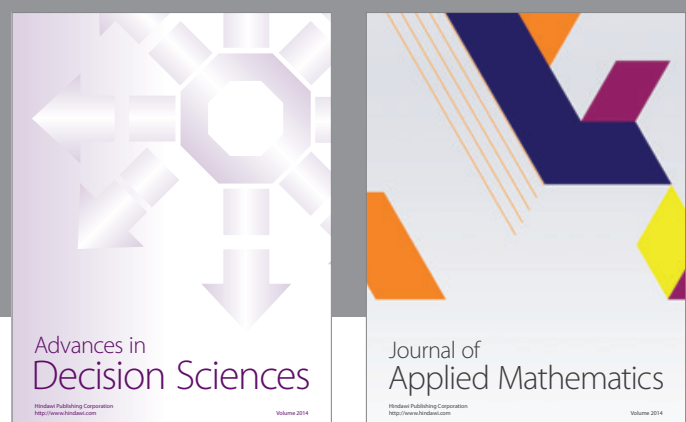

Journal of

Applied Mathematics
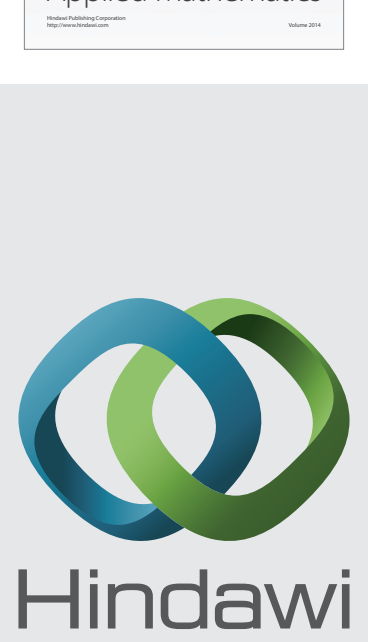

Submit your manuscripts at http://www.hindawi.com
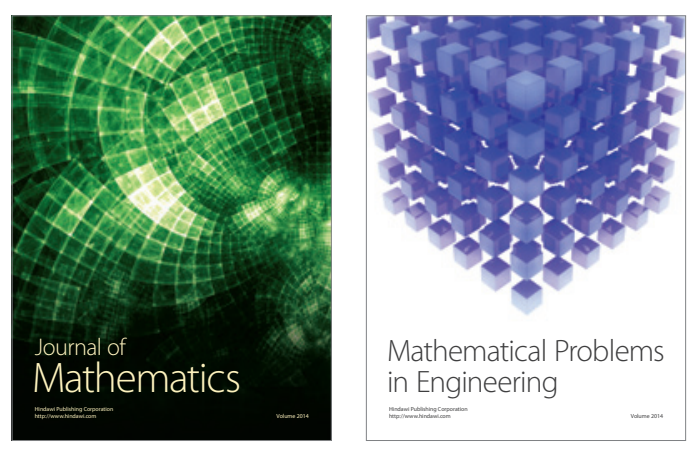

Mathematical Problems in Engineering
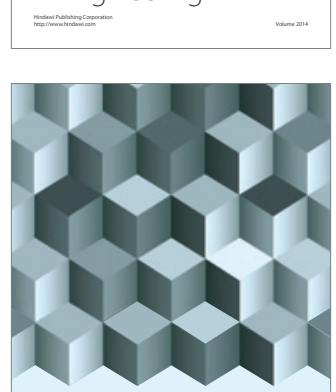

Journal of

Function Spaces
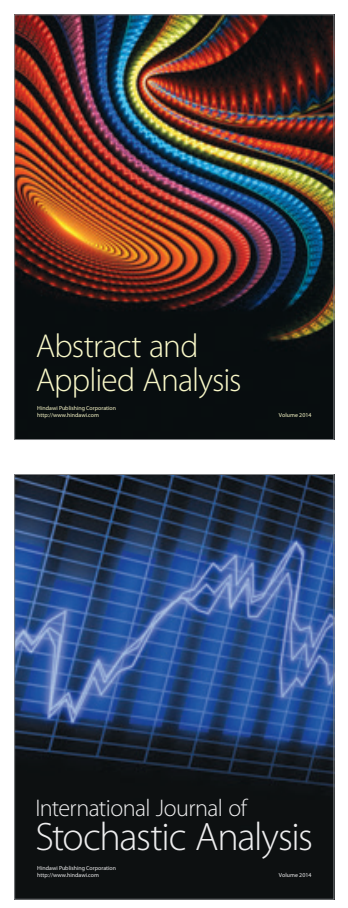

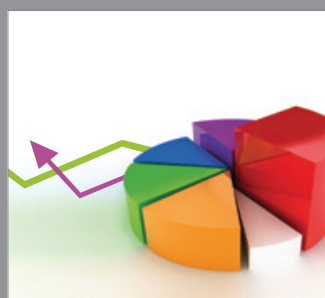

ournal of

Probability and Statistics

Promensencen
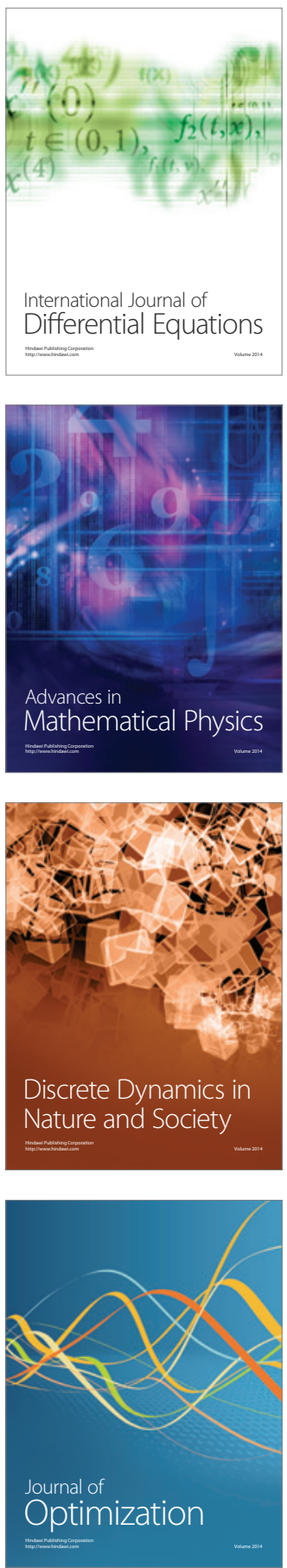